\title{
Entrelacs
}

Cinéma et audiovisuel

Hors-série $n^{\circ} 4$ | 2016

Paysages en séries

\section{Vancouver, lieu de substitution : paysage emblématique/paysage générique.}

Vancouver, lieu de substitution:

Julie Ambal et Aurélien Ramos

\section{(2) OpenEdition}

Journals

Édition électronique

URL : http://journals.openedition.org/entrelacs/2153

DOI : 10.4000/entrelacs.2153

ISBN : $2261-5482$

ISSN : 2261-5482

Éditeur

Éditions Téraèdre

Édition imprimée

Date de publication : 1 novembre 2016

ISSN : 1266-7188

\section{Référence électronique}

Julie Ambal et Aurélien Ramos, « Vancouver, lieu de substitution : paysage emblématique/paysage générique. », Entrelacs [En ligne], Hors-série n 4 | 2016, mis en ligne le 06 février 2018, consulté le 19 avril 2019. URL : http://journals.openedition.org/entrelacs/2153 ; DOI : 10.4000/entrelacs.2153

Ce document a été généré automatiquement le 19 avril 2019

Tous droits réservés 


\title{
Vancouver, lieu de substitution : paysage emblématique/paysage générique.
}

\author{
Vancouver, lieu de substitution:
}

Julie Ambal et Aurélien Ramos

1 S'intéresser aux paysages représentés et diffusés dans les séries télévisées, c'est comprendre le lien qui unit le lieu de tournage avec le lieu du récit. Il s'agit là d'une véritable question de paysage. Le paysagiste fonde en effet sa pratique sur le processus itératif qui l'amène à passer de la réalité physique d'un territoire à ses représentations. Dans cette dialectique fondatrice pour la théorie du paysage se joue la dualité moderne entre sujet et objet mais également celle entre individu et collectif, passé et futur. Pour les paysagistes que nous sommes, l'objet d'étude que constitue la série télévisée apparaitt comme un vecteur particulièrement privilégié de diffusion et de fabrication de représentations sérielles de paysage. La série télévisée participe à la création d'une familiarité au fur et à mesure des épisodes avec un paysage qui devient un «paysage quotidien ».

2 Pour analyser l'efficacité de ce type de média en tant que producteur de représentations du paysage, il nous semble important d'étudier la nature des interactions existant entre un territoire et une fiction.

3 Il est très fréquent que les fictions audiovisuelles ne soient pas tournées dans les lieux où se déroule leur action, mais dans ce qui est communément appelé des « substitute locations ", que l'on peut traduire par « lieux de substitution ».

4 Ce procédé permet à des espaces ayant leurs caractéristiques propres d'en incarner d'autres via deux mouvements distincts: la transformation directe par maquillage, masque, décoration, et la transformation indirecte via la post-production, le montage, les incrustations par green screen, et les différents procédés techniques offerts par les effets spéciaux. À ces deux types de transformations s'ajoutent trois catégories de facteurs entrant dans la détermination du choix de ces lieux de substitution: les facteurs 
pratiques, le rapport d'analogie entre le lieu de tournage et le lieu du récit, et enfin la capacité évocatrice d'un espace, son imagibilité (Lynch, 1960:11), soit la constitution d'éléments distincts et reconnaissables.

5 Vancouver et plus largement son État, la Colombie Britannique, représentent un cas à part dans le paysage audiovisuel américain. Troisième ville de l'industrie audiovisuelle nord-américaine après Los Angeles et New York, Vancouver accueille $10 \%$ de la production hollywoodienne.

La liste des films et séries tournés à Vancouver est longue. On peut en citer quelques exemples : X-Men 2 (2003), La Planète des Singes (2014), Mission: Impossible - Protocole fantôme (2011), TRON : Legacy (2011), Juno (2007), I, Robot (2004), Watchmen (2009), Fifty Shades of grey (2015) pour les films; The 100 (The CW, 2014-présent), Fringe (FOX, 2008-2013), Psych (USA Network, 2006-2014), Arrow, (The CW, 2012-présent), The L Word (Showtime, 2004-2009), The 4400 (USA Network, 2004-2007) pour les séries. Les nombreux sites internet et forums qui se sont donnés pour mission de recenser des lieux de tournage ou des informations sur les tournages en cours dans Vancouver et sa région, attestent de cette activité cinématographique prolifique ${ }^{1}$. Si la plupart de ces plateformes sont animées par le public et les différentes fandoms des séries tournées dans la région, la ville de Vancouver s'est appropriée cette caractéristique de son économie locale pour en faire un argument marketing à destination du tourisme ${ }^{2}$.

7 La ville représente aujourd'hui 6 M.\$CAN de l'industrie du cinéma et des séries télévisées et emploie 125000 personnes. Elle absorbe 59\% des films et séries américaines tournées au Canada (Arnoult, 2015).

Différents facteurs pratiques peuvent l'expliquer, notamment une culture commune en Amérique du Nord, une même langue et une facilité de circulation entre les deux pays ; ou encore une proximité géographique avec le géant de l'industrie cinématographique et télévisuelle qu'est Los Angeles, située sur le même fuseau horaire. À cela s'ajoute le coût de la main-d'œuvre qui est inférieur au Canada, ainsi que la faiblesse du taux de change du dollar canadien. Enfin, des avantages fiscaux attractifs non négligeables : la ColombieBritannique offre $33 \%$ de crédit d'impôt aux productions cinéma et télévisuelles.

9 Il faut maintenant présenter les caractéristiques du paysage de Vancouver et de sa province. La ville se situe sur la péninsule Burrard dans les basses-terres de la ColombieBritannique, sur la côte ouest du Canada. Au sud, se trouve le fleuve Fraser, à l'ouest l'île de Vancouver qui protège la ville du Pacifique et, en limite ouest, les Rocheuses. Au sud, la frontière états-unienne et le Mount Baker constituent l'horizon de la ville.

La végétation est caractérisée par la flore des forêts tempérées humides. De nombreuses espèces endémiques d'Asie se sont implantées au gré des migrations. On peut alors trouver, dans Vancouver, des cerisiers du Japon comme arbre d'alignement, mais aussi des Araucarias du Chili, des azalées ou des magnolias.

Le développement urbain a été fulgurant au XXème siècle, l'architecture verticale s'intensifiant à partir des années 1950. Cette ville est marquée par son patrimoine architectural victorien (fin XIXème siècle) et édouardien (XXème siècle) puis par son développement au XXème siècle.

Le central business district (CBD) est extrêmement dense avec une architecture Moderniste. De grands parcs publics comme le Stanley Park ont été aménagés. Le port industriel, moteur de l'économie de la ville, se situe en son cœur, entre sa partie sud sur la péninsule Burrard et la baie du même nom au nord. L'architecture est marquée par des influences 
new-yorkaises et son urbanisme par le modèle de Hong-Kong (Douay, 2015). Vancouver est une métropole qui illustre une hybridité urbaine très forte.

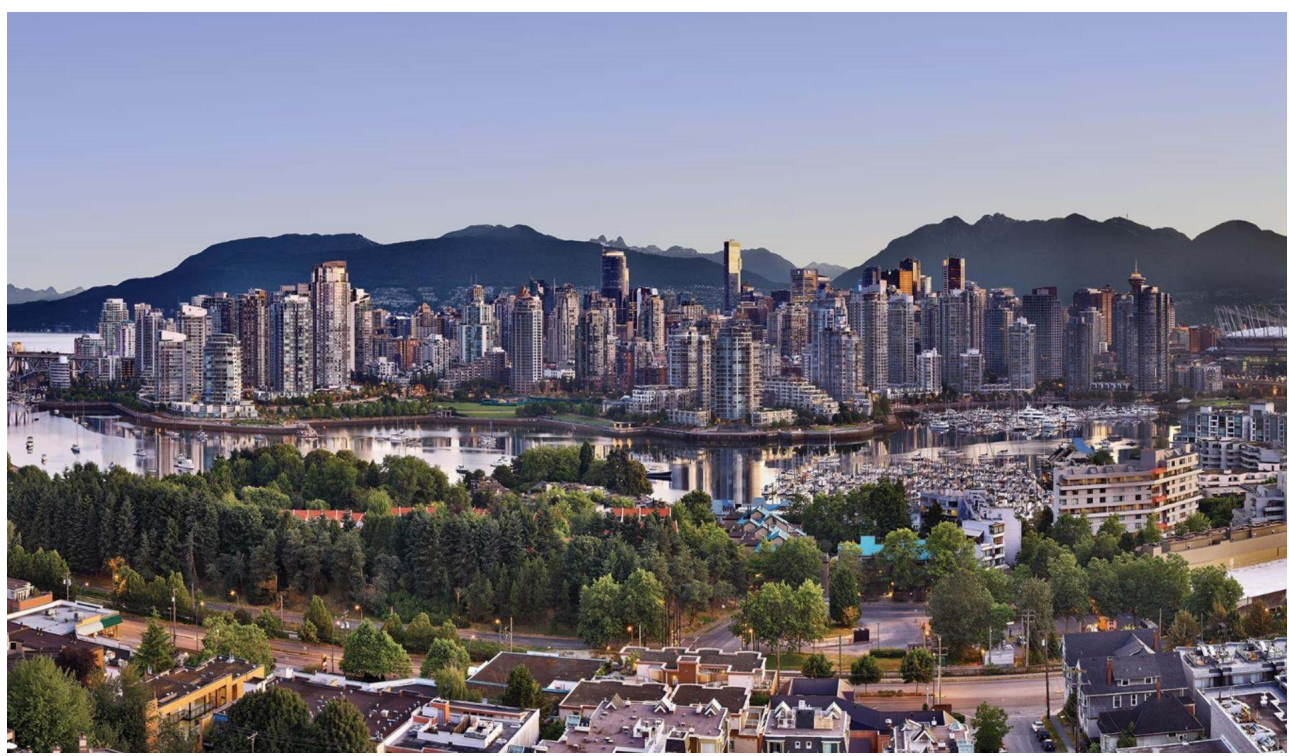

Fig. 1 Vue Vancouver www.tourismvancouver.com

Ce territoire est donc peu neutre, autant en termes géographiques que sur le plan de son caractère urbain. Au-delà des facteurs économiques et fiscaux, dans quelles mesures les caractéristiques singulières de Vancouver participent-elles de son succès comme lieu de substitution? Au vu de la diversité des productions qui s'y déroulent et de la diversité des sites que la ville incarne à l'écran, on peut s'interroger sur les autres critères entrant en jeu. Comment expliquer la disponibilité et la flexibilité de Vancouver en tant que lieu de substitution? Au travers d'un corpus de séries télévisées tournées à Vancouver, nous interrogerons la production par cette ville de modèles génériques de paysages, exportables et mondialisés. Il s'agira d'éclairer la nature de ce caractère générique du paysage de Vancouver et de son impact à la fois sur les représentations mais également, en retour, sur le paysage de Vancouver lui-même.

14 La phase exploratoire de cette étude a consisté à visionner des épisodes de séries tournées à Vancouver afin de pouvoir constituer une première typologie de relations entre monde fictionnel et monde réel. Nous avons déterminé un corpus de séries dans lesquelles le paysage urbain jouait un rôle dans la fiction. Nous avons donc exclu toute série se déroulant exclusivement en intérieur. Au sein de ce corpus, nous avons identifié six types de relations entre le monde réel, Vancouver, et le monde fictionnel. La première relation est la plus évidente : Vancouver incarne Vancouver, la série Continuum en constituant un rare exemple. Le deuxième type de relation entre monde fictionnel et monde réel concerne les séries où Vancouver incarne une ville ou toute la région du nord-ouest américain (Life Unexpected). Le troisième type concerne les séries où Vancouver incarne n'importe quelle ville en Amérique du nord: c'est le cas dans les séries The X-Files, Supernatural ou encore Fringe. En poussant ce schéma, on peut identifier un quatrième type de relation relative aux séries qui prennent le paysage de Vancouver pour représenter toutes les métropoles du monde. Vancouver est aussi le lieu de tournage de séries situant leur action dans des mondes imaginaires. Elle peut alors incarner tour à tour une ville imaginaire en Amérique (The Flash, Arrow, Smallville) ou ailleurs dans la galaxie (Battlestar Galactica). 


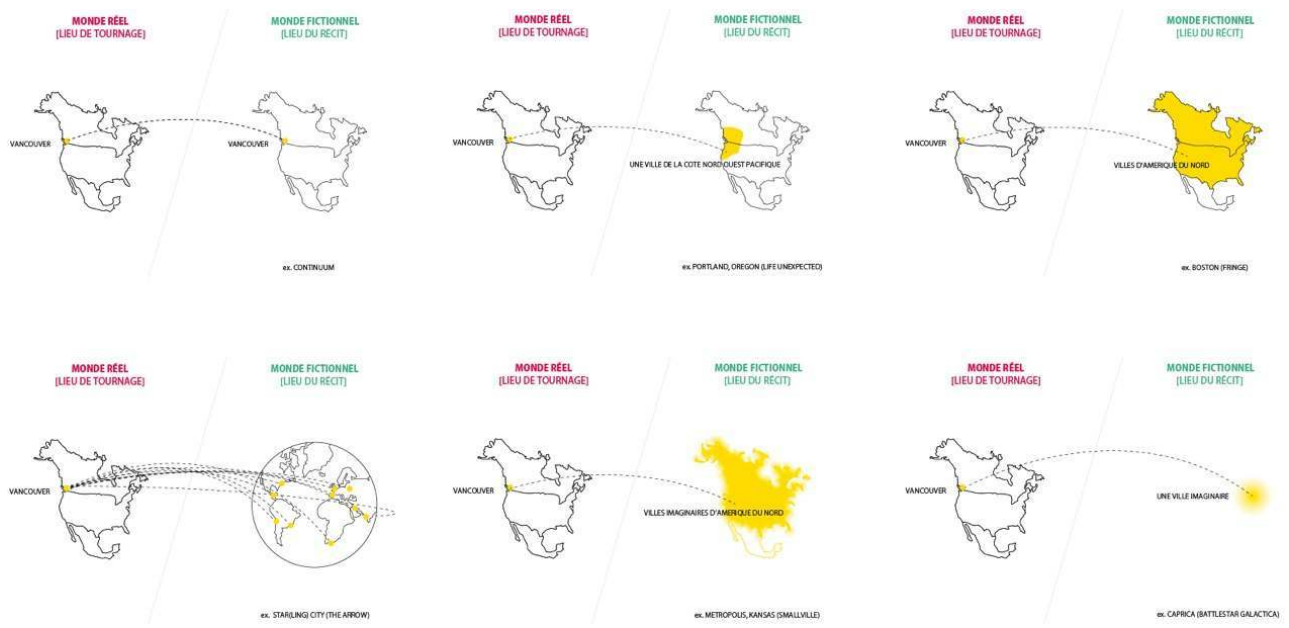

Fig. 2 Schémas monde fictionnel monde réel

Nous allons tenter d'illustrer ces types de relation au travers de trois catégories de rôles joués par le paysage de Vancouver dans les séries télévisées.

\section{Vancouver, base de décor}

La première catégorie concerne les séries utilisant Vancouver comme lieu de tournage, sans exploiter les caractéristiques de son paysage urbain. Il s'agit de séries telles que Human Target où elle incarne San Francisco, Rogue dont l'action principale se déroule à Oakland, ou encore Mistresses, série tournée à Vancouver à partir de la troisième saison, dont l'histoire se situe à Los Angeles. C'est cette dernière série que nous nous proposons d'étudier ici. Dans Mistresses les décisions économiques et pratiques sont à l'origine du choix de Vancouver comme lieu de tournage. Cependant, le paysage urbain ne se traduit nullement dans le monde fictionnel. La ville offre un simple support physique dont les rares détails identifiables sont minutieusement effacés ou détournés. Vancouver peut alors incarner indifféremment une métropole aux antipodes d'elle-même et du caractère de son paysage urbain, telle que Los Angeles.

Dans cette série, le cadre spatial fictionnel est évoqué de différentes manières : par des vues cadrées sur des édifices architecturaux pour les establishing shots ${ }^{3}$, par des vues urbaines au cadre serré en travelling, par des scènes en intérieur, essentiellement tournées en studio et utilisant des incrustations pour matérialiser les vues extérieures par la fenêtre.

Dans le premier cas, les édifices donnés à voir se caractérisent par leur banalité mais présentent des détails architecturaux à la marge indiquant le cadre géographique suggéré : la maison individuelle en parement bois et bow-windows pour les suburbs de San Francisco, Boston ou Washington ; la maison de style côtier, avec sa terrasse et ses baies vitrées pour évoquer les banlieues de Los Angeles et de la côte Ouest par exemple. Dans le deuxième cas, le cadre urbain qui apparaît en arrière-plan des personnages (lorsqu'il n'y a pas d'incrustation bien sûr) se caractérise également par sa banalité. Le paysage urbain est marqué par la domination de la voiture, des infrastructures et de la signalétique qui l'accompagne, c'est la ville franchisée telle qu'elle est décrite par David Mangin (2014). Apparaissent également les paysages de suburbs résidentiels caractérisés par leurs 
maisons individuelles bordant une chaussée sinueuse aux larges trottoirs, et leurs front gardens richement fleuris.

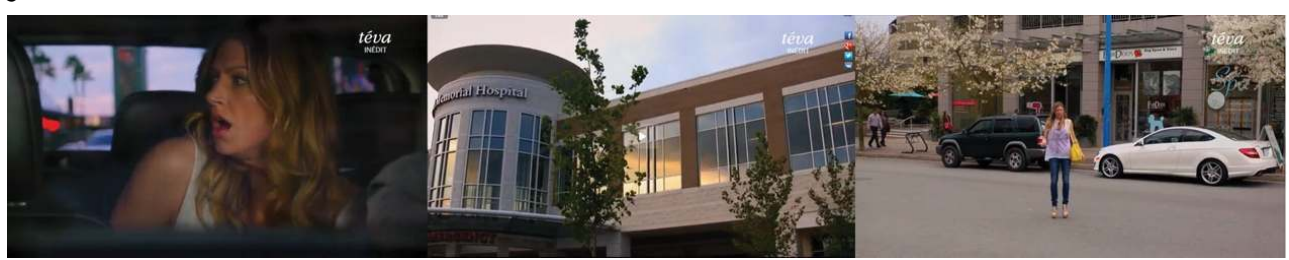

Fig. 3 Mistresses

19 L'incarnation par Vancouver de Los Angeles et plus précisément de Beverly Hills constitue un cas intéressant de neutralisation du contexte réel. Mike Davis a défini Los Angeles, comme une « ville-symbole » (1998), une ville bâtie à l'échelle internationale qui fonde son identité sur son absence d'image locale. Los Angeles peut alors être incarnée à très peu de frais, par n'importe quel autre cadre urbain. Dans Mistresses, l'ajout d'indices climatiques - palmiers dans les rues, signalétique indiquant des lieux identifiables, vêtements des personnages - permettent de maquiller Vancouver en ville californienne. Dans cette catégorie, le paysage urbain joue un rôle purement secondaire. Moins qu'indicatif, il constitue parfois même un obstacle physique à dépasser, maquiller. C'est un simple support spatial à transformer selon les besoins de la fiction.

\section{Vancouver, hyperbole d'une région}

21 Une deuxième catégorie de séries se dessine pour laquelle le cadre spatial et le paysage urbain jouent un rôle important. Il s'agit de séries dont l'action se déroule dans un cadre géographique proche de Vancouver, généralement de l'autre côté de la frontière, dans l'État de Washington ou dans l'Oregon. Nous évoquerons ici plus particulièrement les cas de trois séries tournées à Vancouver, Life Unexpected dont l'action se situe à Portland, The 4400 et The Killing se déroulant à Seattle.

Dans ces séries, la représentation des villes de la fiction prend appui sur des establishing shots en travelling aériens sur des vues de la ville fictionnelle. Ces vues fixent les landmark (Lynch, 1960 (1999) : 92), des paysages urbains ou des éléments de paysage qui constituent des marqueurs pour tout un territoire et fabriquent une identité efficace et reconnaissable au fil des épisodes. Répétés à maintes reprises au fil des épisodes comme transition entre deux scènes, ils inscrivent dans la mémoire du spectateur les informations nécessaires à l'identification du cadre spatial de la fiction. À ces vues réelles tournées sans acteurs dans la ville de la fiction s'ajoutent les scènes tournées à Vancouver. Pour ces séries, les similitudes géographiques et paysagères de Vancouver avec les lieux de la fiction constituent un avantage qui est exploité pour les besoins de la série. Tout d'abord, le climat océanique et pluvieux qui caractérise ces territoires du nord-ouest américains renforce l'identification régionale. Cela se traduit par la similitude de l'identité végétale, celle des forêts tempérées humides. 


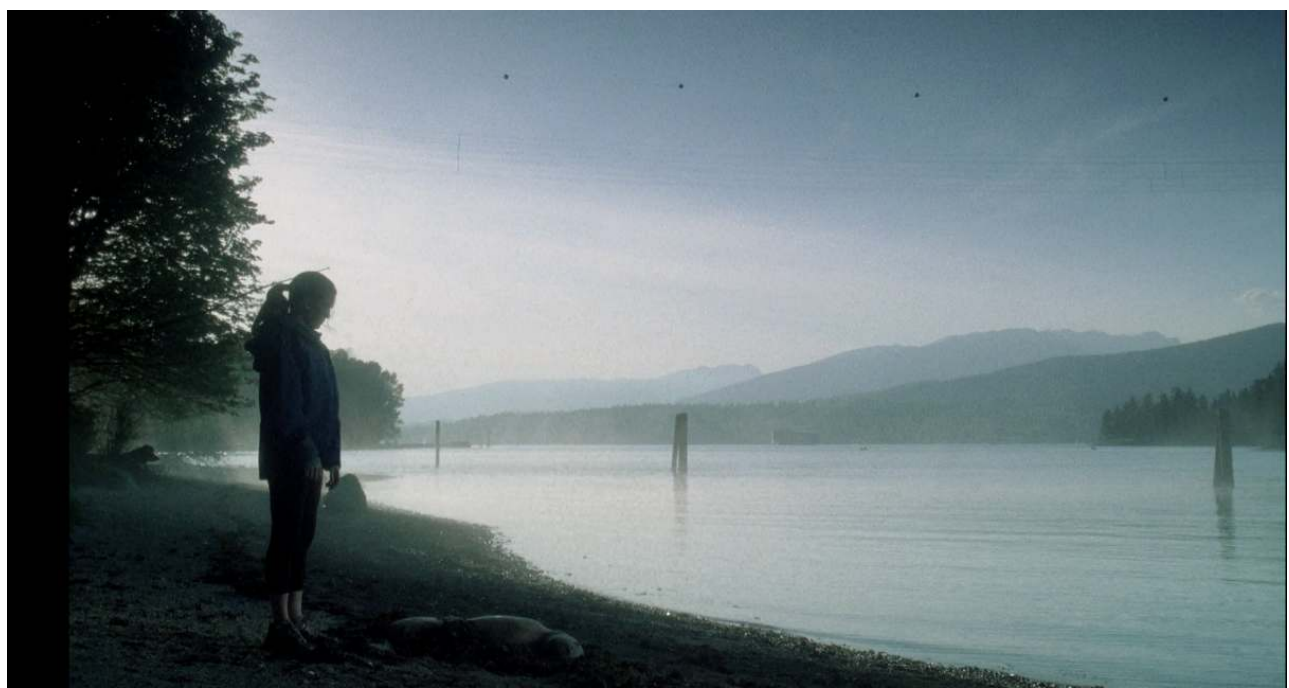

Fig. 4 The Killing - Pilot

La présence des Rocheuses comme fond de scène du paysage urbain associée à celle de la côte et d'un bras de mer permettent, par mimétisme, d'évoquer avec la baie de Vancouver le Puget Sound voisin et la baie de Seattle.

Enfin, le caractère portuaire et industriel de Vancouver permet d'évoquer facilement celui de Seattle et de Portland. Les trois villes se caractérisent en effet par leur développement industrialo-portuaire à l'embouchure d'un fleuve.

Mais le plus intéressant concerne les prises de vues en extérieur, réalisées à Vancouver mais représentant Seattle ou Portland. Si le paysage urbain associé à l'identité géographique et aux indices climatiques suffit à suggérer une de ces villes, le simple ajout de quelques signaux explicites et de détails urbains (marques, signalétique, publicités) permet en un coup d'œil d'affirmer une identité.

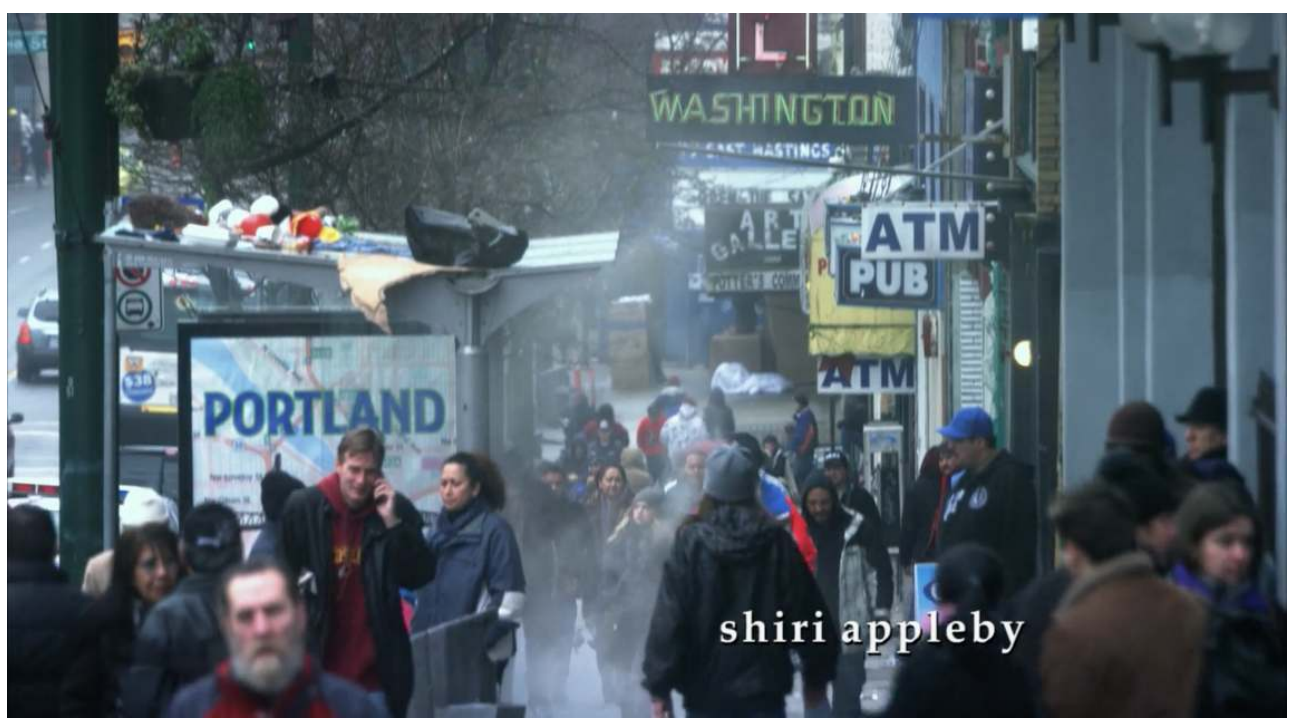

Fig. 5 Life unexpected - Pilot

La ville est dans ces séries un personnage à part entière. Vancouver devient un véritable paysage emblématique, cristallisant en un seul et même lieu toutes les caractéristiques spatiales d'une région transfrontalière. Ces séries contribuent à instituer Vancouver et la région nord-ouest pacifique - à l'instar des villes californiennes, ou plus encore de New 
York - comme un paysage mythique de la fiction nord-américaine. Ce, à la différence près qu'ici Vancouver symbolise et incarne plus qu'elle-même: ses représentations la dépassent largement. L'analogie est poussée jusqu'à l'exagération.

L'analyse de cette catégorie de séries dans lesquelles Vancouver incarne d'autres territoires existants ou des sites fictionnels dans un cadre géographique réel met en lumière un paradoxe: Vancouver n'est pas Los Angeles, ce n'est pas une ville-symbole construite pour le monde mais coupée de son ancrage local. Il ne s'agit pas d'une ville sans identité locale. Pour preuve, ses caractéristiques géographiques et climatiques, ses landmarks, sont exploités pour signifier une ambiance paysagère régionale. Pourtant, elle est également suffisamment générique et anonyme pour être détournée et transformée afin d'incarner ce qu'elle n'est pas.

Vancouver semble jouer de l'ambivalence simultanée entre une imagibilité paysagère forte et une imagibilité urbaine faible, et l'un comme l'autre sont interdépendants. Il faut alors s'interroger sur la nature de ce caractère générique.

\section{Incarnation d'un modèle générique}

La dernière catégorie de séries expose un caractère générique plus ou moins fort qui va incarner plusieurs modèles. Le premier est l'incarnation de paysages américains, un paysage générique, ordinaire, quotidien, sans images fortes de l'Amérique au travers de deux séries : Supernatural et The X-Files.

Le deuxième modèle révèle un paysage générique d'unité " paysagère » qu'on pourrait appeler « cliché » : la figure avec une universalité recherchée sans un caractère localisé.

31 Enfin le modèle par excellence de ville mondialisée capitaliste commune (Arrow, The Flash, Smallville) qui incarne un paysage urbain « typique », voire emblématique.

\section{Incarnation des paysages américains}

Dans la série Supernatural, la fiction trouve dans Vancouver le cadre d'incarnation parfait : en effet, la série met en scène la dérive des deux frères Winchester au travers de l'Amérique, traquant les phénomènes surnaturels qui peuplent les croyances et mythes populaires. Leurs enquêtes, qui les amènent également aux quatre coins des USA, ont principalement pour scène la "small town america » (Elisabeth Tovar), les villes moyennes américaines sans caractère ; le "lost continent » comme le décrit l'écrivain américain Bill Bryon, ces petites villes qui génèrent peu d'urbanité. Un décor fait de motel, diner, camping caravaning, bar glauque, entrepôts, cimetières, usines désaffectées, mines et stations essences. Ici, le but recherché est de faire le portrait de cette Amérique oubliée, sans identité, loin des fortes images et représentations mondialisées des USA. Vancouver se présente alors comme le meilleur décor possible, offrant un cadre urbain à l'architecture générique, des espaces périphériques périurbains anonymes.

Dans The X-Files, au fil des saisons, les enquêtes de Mulder et Scully les entraînent aux quatre coins du pays. Le siège du FBI et de l'action se situent à Washington DC sur la côte Est. Pourtant l'intégralité des 5 premières saisons a été tournée à Vancouver. Ainsi, le paysage de Vancouver sert aussi bien à incarner New York (S01E11) que le Chinatown de San Francisco (S03E19), Dallas ou Memphis (S04E07) et même une base militaire à San Diego (S05E06). 

même espace incarnant toujours la même catégorie d'espace, produisant une figure paysage. voisines permet de signifier à moindres frais et sans trop de maquillage :

- Des suburbs résidentiels très arborés, des maisons en parement bois, avec bow-windows de style victorien, Queen Anne ou Art and Craft.

- Des faubourgs industrialo-portuaires et très décrépis (le quartier Vancouver Eastside ou Gastown sont maintes fois convoqués), architecture néo-romane à briques.

- Un CBD à l'architecture internationale, lieu de travail et de consommation (buildings vitrés, centre-commerciaux).

Les bâtiments institutionnels sont simplement réexploités dans la série soit en gardant leur fonction mais en changeant leur nom ou leur localisation soit en les détournant de leur fonction pour en incarner une autre assez proche. Par exemple, la récurrence des sites universitaires SFU et UBC, ou encore l'utilisation de la Vancouver Art Gallery, ancien Palais de Justice, pour incarner un véritable palais de justice sont de simples déplacements sémantiques. musée, une agora et un marché. 


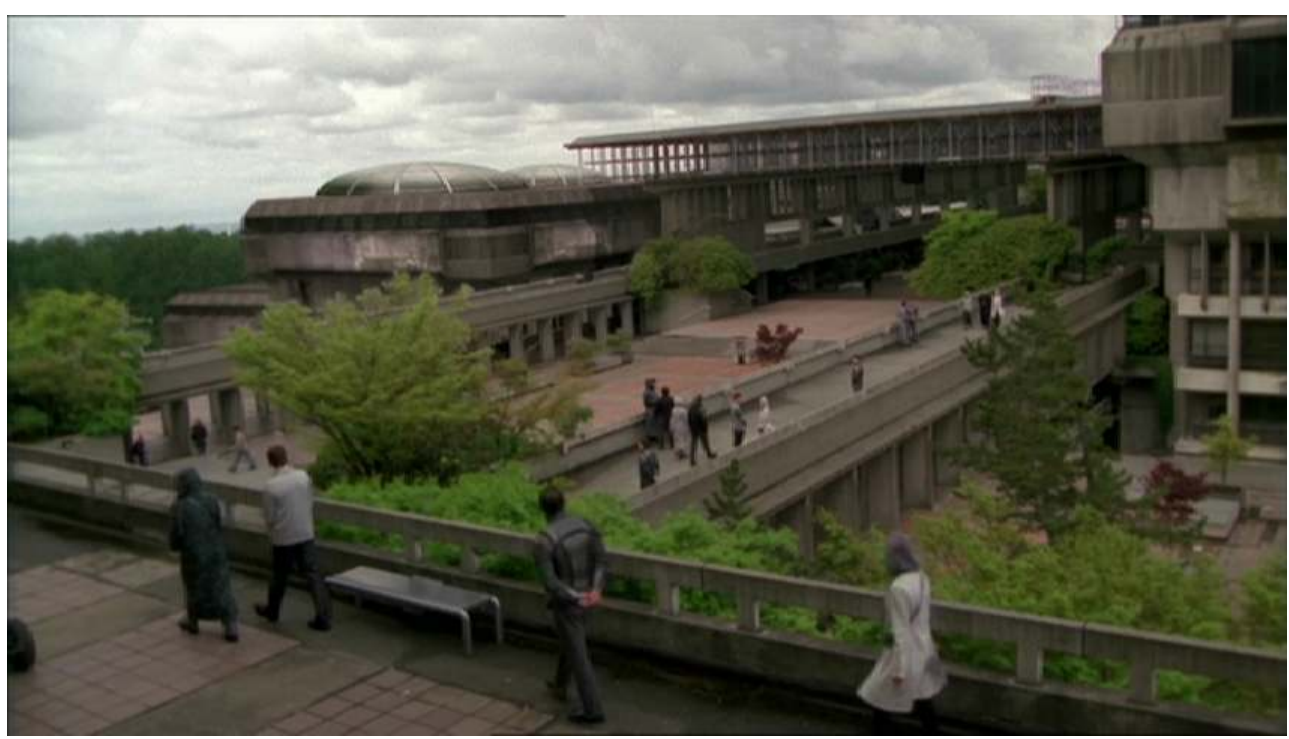

Fig. 6 Stargate SG-7, Tollana City S03E15, sur le site de la SFU

Dans un deuxième temps, nous examinons les séries qui s'appuient ou exploitent des figures "paysagères emblématiques » pour véhiculer des valeurs. Les unités paysagères qui s'élèvent comme modèles dans les séries ont le caractère générique de leur forme mais ont aussi une valeur d'universalité. Ces modèles génériques n'ont pas besoin d'être situés dans le récit, leur forme exprime un imaginaire très fort et détermine une localisation floue mais universelle; par exemple la figure de suburb se situe systématiquement en dehors du cœur de métropole ou de ville, dans une périphérie plus ou moins éloignée mais protégée de tous les désagréments que produit l'hyper-urbain. Par ailleurs, ces deux types de paysages sont souvent en opposition dans l'histoire, l'ambiance de la suburb est souvent représentée avec des lumières chaudes, il y fait beau, il y a de la végétation, les rues sont calmes et peu encombrées alors que la ville est beaucoup plus complexe, emplie d'obstacles, l'ambiance y est plus froide, dans des tons bleus, noirs, rehaussée par de nombreux points de lumière blanche.

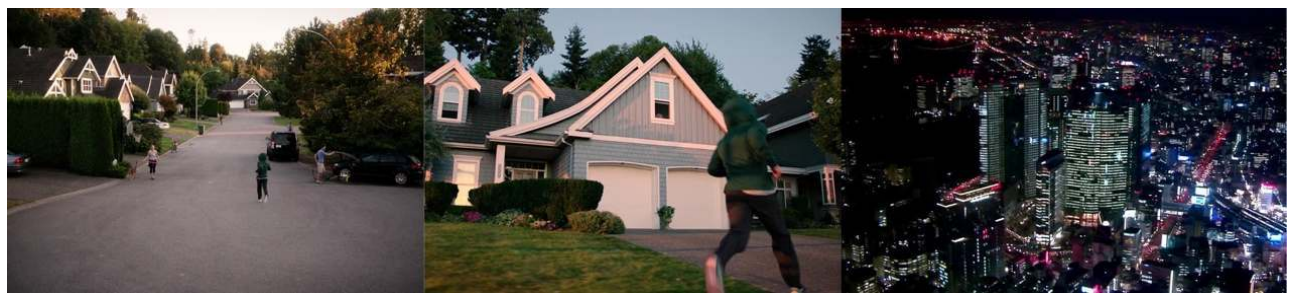

Fig.7 Arrow S04E01

46 En sortant de la métropole, le territoire de Vancouver offre aussi un paysage de «nature » que les séries se réapproprient pour évoquer tous types d'unité paysagère 
générique : le paysage insulaire sauvage (Arrow), le paisible ranch (Smallville) ou la forêt ( Arrow, Battlestar Galactica S01E01, S02E09, S02E17).

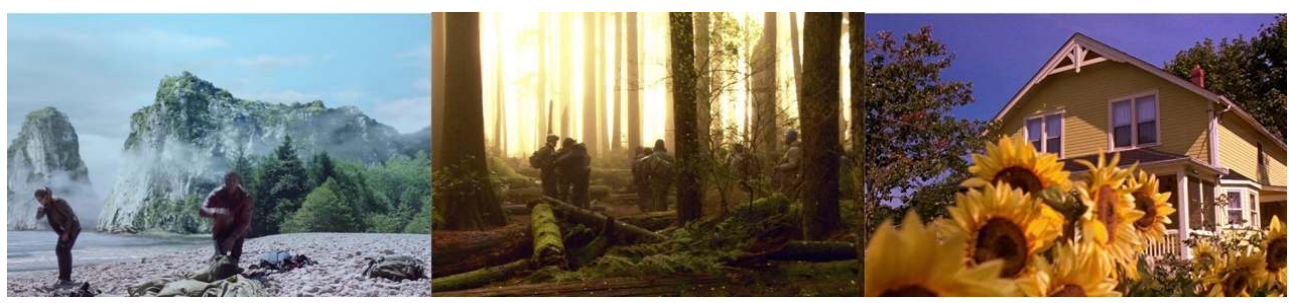

Fig. 8 île, forêt, ranch

\section{Le modèle par excellence de ville mondialisée commune, modèle universel}

\section{Les cas de DC Comics et de Continuum}

Dans les séries adaptées de l'univers de DC Comics, les paysages représentés sont situés dans des villes et/ou métropoles d'un côté générique, car reprenant le modèle de la ville mondialisée par excellence mais avec souvent un emblème, un landmark qui représente une idée, une marque imaginée mais reconnaissable de la métropole complexe et unique.

Rem Koolhaas dans un texte éponyme pose les fondements d'une critique de la ville mondialisée (1994). Ville de la postmodernité, la ville générique pour Koolhaas est d'origine américaine. C'est une ville fractale, reproductible à l'infini, ville anomique qui s'est affranchie du sol et affranchie des frontières. Une ville qui a évacué la notion d'identité pour devenir multinationale. La ville générique possède cependant un passé, traité comme alibi: l'histoire se matérialise sous forme de prestations touristiques, comme industrie de la production de souvenirs symboliques et monnayables. Il y a donc toujours un ancien quartier industriel et toujours un port ou un fleuve, un élément géographique omniprésent et reproduit en série sous forme d'emblème ou de logo. La ville générique a transformé l'identité spatiale en mantra.

Vancouver est un exemple parfait illustrant la définition de généricité vu par Koolhaas et c'est ce caractère qui la rend si convoitée pour tourner des séries comme celles de superhéros qui se situent dans des univers parallèles, en faisant référence à des aspects de notre monde réel comme dans l'univers de DC Comics. Nous parlerons ici de Smallville, Arrow et The Flash.

\section{Métropole générique mondialisée}

Dans les séries Arrow et The Flash leurs villes respectives, Starling City et Central City, sont de grandes métropoles taillées dans un modèle principalement nord-américain mais qui pourrait représenter une métropole partout dans le monde.

Les traits classiques évoqués par la ville générique de Koolhaas sont repris par ces séries : plan orthogonal (The Flash, générique), un CBD avec ses tours de building vitrées (Arrow), une ville très éclairée de nuit (Arrow), de grands équipements comme le cimetière ou la prison (Arrow, The Flash), un port et ses docks industriels, des rues larges avec commerces et taxis, une emprise viaire importante avec beaucoup de flux, un quartier populaire appuyé (souvent très pauvre) avec des SDF et des hangars industriels abandonnés. 


\section{Emblème, marque, landmark} et par elle-même. Elle permet plutôt d'en signifier une " autre » : un autre lieu, un autre monde. Vancouver, représenté dans Continuum, joue finalement au même jeu et se personnifie par l'utilisation de ce modèle généralisé, en reprenant par exemple la même allée ou la même rue que les séries citées dans cette communication.

\section{Vecteur de diffusion ou production de modèles urbains génériques?} d'un public mondialisé. Il existe donc une relativité dans ces représentations puisqu'elles ne valent que pour un type particulier de public, non un public local en capacité de reconnaître les lieux réels. Les séries misent sur une certaine méconnaissance des paysages représentés pour permettre à la fiction de trouver place. fabrquent des représentations urbaines génériques de ce qu'est la métropole nordaméricaine, voire mondiale. La série devient un formidable vecteur de diffusion d'un modèle générique.

Prenons un exemple de la relativité de représentation à destination des publics. Une scène dans la série Life Unexpected fait référence à une rue qui n'existe pas à Portland mais bien à Vancouver. Nous l'avons recherché sur google maps et nous avons retrouvé le lieu de tournage à Vancouver : la signalétique des rues de Vancouver n'est pas changée sur la

Entrelacs, Hors-série $n^{\circ} 4$ | 2016 
capture d'écran de la série. On peut affirmer qu'il existe un gradient de généricité : pour un téléspectateur autre que nord-américain, la méconnaissance de la situation entraîne une adhésion à la fiction; pour un téléspectateur américain il existe une certaine familiarité et une probabilité que le lieu de tournage soit aussi le lieu de l'histoire ; mais pour un téléspectateur de Portland ou de Vancouver, il doit exister une familiarité avec leur ville qui leur révèle que ce lieu ne peut pas se trouver dans la ville de l'histoire.
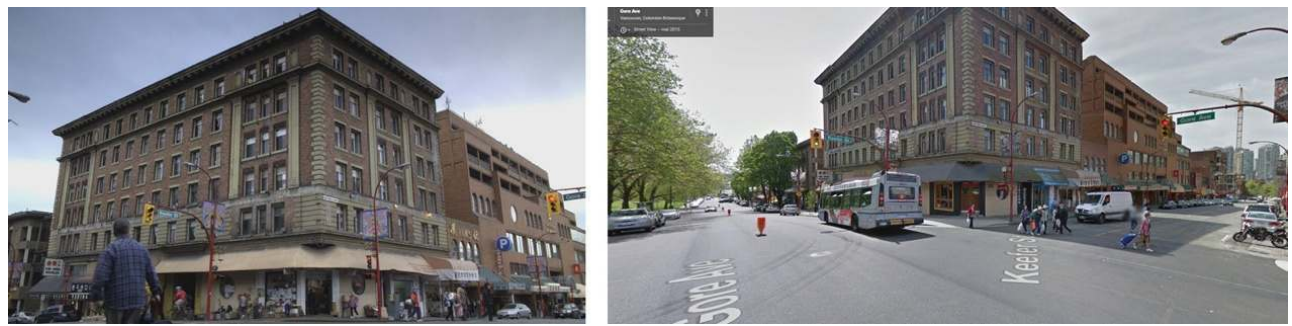

Fig. 9 Keefer Str dans Life Unexpected

61 Pourtant, si une certaine méconnaissance locale est nécessaire, les séries télévisées génèrent un public qui acquiert une certaine expertise au sein du cadre fictionnel des séries. Ainsi, les fans deviennent des experts dans la reconnaissance et l'analyse spatiale des espaces représentés : ils reconstituent une géographie du territoire américain fragmentaire. Cette expertise de l'espace fictionnel met en lumière la plasticité fictionnelle des espaces représentés.

Pour le métier de paysagiste, qui est un métier encore en voie de définition, nous pouvons en retirer l'importance de la responsabilité de la fiction dans la fabrication de représentations d'espace. Le récit, le discours et la mythologie fabriquent des espaces pour lesquels le public (mondialisé), épisode après épisode, constitue des représentations.

\section{Bibliographie, webographie}

Arnoult, Frédéric. «Les caméras américaines tournent à plein régime au Canada », radio-canada.ca , 27.04.15. Repéré à

http://ici.radio-canada.ca/nouvelles/economie/2015/04/27/002-industrie-cinema-canadacredits-impot-faiblesse-dollar.shtml, consulté le 19 avril 2016.

Begout, Bruce. Lieu commun. Paris : Éd. Allia, 2003.

Begout, Bruce. Suburbia. Paris : Éd. Inculte, 2013.

Cadiou, Nathalie et Lugingühl, Yves. « Modèles paysagers et représentation du paysage en Normandie-Maine » in Vois Enat, Claudie (dir.), Paysage au pluriel, pour une approche ethnologique des paysages. Paris : Éd. de la Maison des sciences de l'homme, 1995.

Davis, Mike. City of Quartz - Los Angeles capitale du futur. Paris : La découverte, 1998 (2000).

Davis, Mike. Au-delà de Blade Runner : Los Angeles et l'imagination du désastre. Paris : Éd Allia, 2006.

Douay, Nicolas. « Le Vancouverism : hybridation et circulation d'un modèle urbain », Métropolitiques, 11.03.15. Repéré à http://www.metropolitiques.eu/Le-Vancouverismhybridation-et.html, consulté le 30 avril 2015.

Lamizet, Bernard, Samson, Pascal. Les langages de la ville. Marseille : Éd. Parenthèses, 1997.

Lynch, Kévin. L’image de la cité. Paris : Dunod, 1960 (1999). 
Koolhaas, Rem. La ville générique. 1994. Repéré à http://th3.fr/imagesThemes/docs/ th3_villien_texte_rem_koolaas_la_ville_generique.pdf?PHPSESSI\%

20D=fvtkroupr85hv7al26dvbf2a85, consulté le 08 janvier 2016.

Pavel, Thomas. Univers de la fiction. 1988. pp. 76 et suivantes.

Robin, Régine. « L'après-ville », Communications, 2009, Volume 85, Numéro 1, pp. 185-198.

Sgar, D. Anne. « Entre l'eau, l'arbre et le ciel. Figures paysagères suédoises et construction de l'identité nationale. ", in Géographie et cultures, $n^{\circ} 66$, « Le patrimoine naturel entre culture et ressource », 2008. p. 121 à 138.

\section{Sériographie}

Arrow, The CW, 2012-présent.

Battlestar Galactica, Syfy Channel, 2004-2009.

Continuum, Showcase, 2012-2015.

Human Target, Fox CTV, 2010-2011.

Life Unexpected, The CW, 2010-2011.

Mistresses, ABC, 2013-2016. (tournage à Vancouver à partir de la saison 3)

Rogue, Audience network, 2013-présent.

Smallville, The WB, The CW, 2001-2011.

Stargate SG-1, Showtime, Syfy Channel, 1997-2007.

Supernatural, The WB, The CW, 2005-présent.

The 4400, USA network, 2004-2007.

The Flash, The CW, 2014-présent.

The Killing, AMC (2011-2013), Netflix (2014), 2011-2014.

The L World, Showtime, 2004-2009.

The X-files, Fox, 1993-2002. (5 premières saisons tournées à Vancouver)

\section{NOTES}

1. Liste non exhaustive: http://www.whatsfilming.ca/ ; http://yvrshoots.com/ ; http:// www.filmingvancouver.com/

2. http://www.tourismvancouver.com/activities/sightseeing/filmed-in-vancouver/ : le site de la ville de Vancouver dédié à ce type singulier de tourisme que Matt Hills dans son ouvrage Fan Cultures nomme « cult geography ». Pour poursuivre la réflexion sur le rapport entre le tourisme et la fiction, voir Peyron, David. "L'espace des fandoms part.1, le tourisme de l'imaginaire ", David Peyron, 03.06.16. Repéré à https://davidpeyron.wordpress.com/2016/06/03/lespace-dufandom-partie-1/, consulté le 7 juillet 2016. 
3. Dans le cas de la série Mistresses, de nombreux establishing shots sont issus de banques d'images de vidéos libres de droits, vendus en ligne sur des sites grand public tels que http:// www.gettyimages.fr.

\section{AUTEUR}

\section{JULIE AMBAL ET AURÉLIEN RAMOS}

Julie Ambal est en troisième année de doctorat, sa thèse se propose d'analyser la mise en récit de la ville du mouvement dans le cadre métropolitain de projets urbains contemporains. L'objectif est de comprendre quel est cet imaginaire de la mobilité aujourd'hui, par quels processus se metil en place, comment est-il relayé et porté par les faiseurs de ville (politique et experts) et enfin comment cet imaginaire est reçu par les différents publics.Aurélien Ramos travaille sur une thèse intitulée « Mode, modèles et systèmes de valeurs esthétiques du paysage ». Il analyse la démultiplication des modèles paysagers depuis le milieu du XXe siècle et sa manifestation dans les choix esthétiques des pratiques paysagistes. Par un état des lieux de la diversité des modes de conception en paysage, son travail vise à mettre en lumière les différents systèmes d'influences, d'imitation et de distinction dans les processus d'aménagement de l'espace. 